\title{
Semi-automatic Epileptic Hot Spot Detection in ECD Brain SPECT Images
}

\author{
Laszlo Papp, Maaz Zuhayra, Eberhard Henze \\ Department of Radiology and Nuclear Medicine, UK-SH Campus Kiel, Kiel, Germany \\ lpapp@nuc-med.uni-kiel.de
}

\begin{abstract}
A method is proposed to process ECD brain SPECT images representing epileptic hot spots inside the brain. For validation 35 ictal interictal patient image data were processed. The images were registered by a normalized mutual information method, then the separation of the suspicious and normal brain areas were performed by two thresholdbased segmentations. Normalization between the images was performed by local normal brain mean values. Based on the validation made by two medical physicians, minimal human intervention in the segmentation parameters was necessary to detect all epileptic spots and minimize the number of false spots inside the brain.
\end{abstract}

\section{Introduction}

Epileptic foci detection is a major field in neuroimaging which is mostly performed by obtaining and comparing an ictal and an interictal ECD brain SPECT image about the patient representing an epileptic event and a normal stage of the brain respectively [1]. Since these images are never acquired at the same time, registration between them is necessary, as well as the normalization of them, due to the different dose and biological uptake represented by these images. While registering the image pairs is generally performed with a high confidence [2], normalization methods can strongly affect the result of the ictal-interictal subtraction [3], since they can erase existing hot spots or generate false positive ones. By the other hand even if the chosen normalization method is desired, the subtraction image might represent positive false spots where a non-epileptic normal brain region was brighter in the ictal image than in the interictal one (Fig. 1). Our goal was to develop a program representing our method for detecting true hot spots and minimizing positive false spots by performing local normalization inside the brain only at epileptic suspicious areas.

\section{Materials and methods}

\subsection{Patient data}

35 epileptic ictal-interictal ECD brain SPECT patient were identified in the Picture Archiving and Communication System (PACS) of our clinic and their raw 
image pairs were collected. The raw data were reconstructed by Geometric Mean (GMA) iterative reconstruction. Conversion of the reconstructed images to Analyze format was performed for being compatible with our program implemented in IDL 7.0

\subsection{Image registration}

Both ictal and interictal images were duplicated, and the grey values of the copied images were down sampled between 0 and 255. For similarity measurement the normalized mutual information (NMI) cost function was calculated [4] in the auto registration algorithm (equation 1). For function minimization Downhill-Simplex method was applied with functional tolerance 0.0001 [5]. The byte scaled ictal $A$ image was the reference of the registration and the byte scaled interictal image $B$ was transformed to find the desired transformation parameters. A rigid transformation (shifting and rotation) determined by the auto registration was performed between the original images to superimpose them.

$$
\frac{(H(A)+H(B))}{H(A, B)}
$$

Where $H(A)$ is the Shannon entropy [4] of the image $A$, and $H(A, B)$ is the Shannon entropy of the joint probabilities of images $A$ and $B$.

\subsection{Normalization and hot spot detection}

Two default threshold values were determined for both $A$ and $B$ images

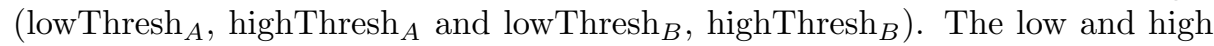
thresh values were $40 \%$ [3] and $75 \%$ of the maximum value of the corresponding image. All voxels in image $A$ higher than highThresh $A$ were marked as epilepticsuspicious areas $\left(E S A_{A}\right)$. All voxels between the corresponding lowThresh and

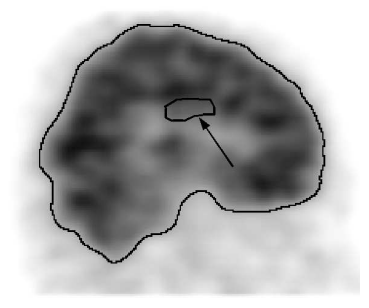

(c) Ictal

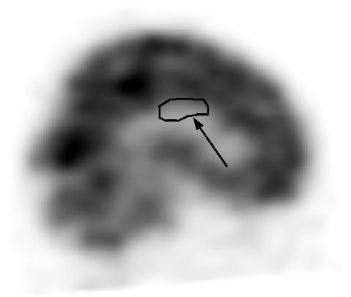

(d) Interictal

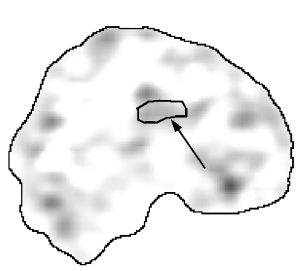

(e) Result of whole brain subtraction

Fig. 1. Sagittal slices of a whole brain subtraction example. The region signed by arrows represents a normal brain area in the ictal image (a). Relatively it has a lower uptake in the interictal image (b) which produces a positive false spot on the subtraction (c). 
highThresh values were marked as normal brain activity areas in both images $\left(N B A_{A}, N B A_{B}\right)$. The mean values $\left(M_{A}\right.$ and $\left.M_{B}\right)$ of $N B A_{A}$ and $N B A_{B}$ were computed for both images respectively. For all $v_{A}$ ictal voxel values inside $E S A_{A}$ a weighting for the given $v_{A}$ voxel value was determined based on equation 2 :

$$
\left(\frac{v_{A}-\operatorname{highThresh}_{A}}{M_{A}}-\frac{v_{B}-\operatorname{highThresh}_{B}}{M_{B}}\right) * v_{A}
$$

where $v_{A}$ and $v_{B}$ were ictal and interictal voxel values respectively at positions defined by $E S A_{A}$ region in both brain images. In the result image all negative values were modified to 0 .

\subsection{Visualization}

A binary image from the ictal image based on lowThresh ${ }_{A}$ was generated, where all values higher than lowThresh $A$ were modified to 1 , other values were modified to 0 . A morphological gradient was performed on the binary image to generate a one voxel thick boundary [6]. The result of the hot spot detection was merged with the boundary image for better visualization and hot spot localization.

\subsection{Validation}

Validation was done by two medical physicians independently. The transformation parameters given by the NMI auto registration was validated in the first step by visual assessment. Modification of the auto-determined transformation parameters was provided to allow necessary corrections. Every manual correction in the NMI transformation parameters was recorded for further registration error analysis. To validate the correctness of the default threshold values the manual threshold modification was provided in the program. For better foci localization the ictal-interictal image pairs, the classic subtraction of them and the result of our method was represented in parallel (Fig. 2).

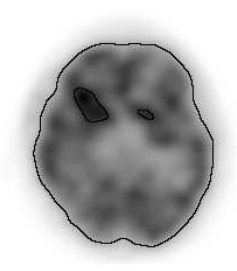

(a) Ictal

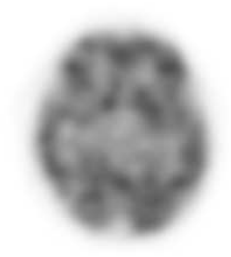

(b) Interictal
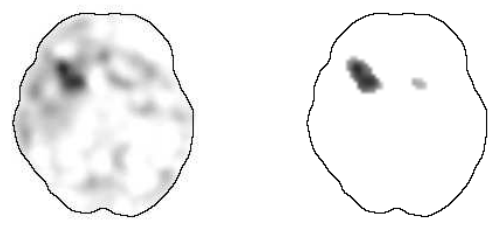

(c) Result of whole (d) Result of our brain subtraction ocal subtraction

Fig. 2. Comparison of the whole brain subtraction (c) with our method (d) on axial slices. Our local filtered result (d) represents hop spots only inside area defined by $\operatorname{highThresh}_{A}$. 


\section{Results}

The average registration error was $1.2 \mathrm{~mm}$ of shifting and 0.37 degrees of rotation. Modification of the auto registration-given transformation parameters was necessary in 2 cases, where the misregistrations were between $2-4 \mathrm{~mm}$ for translation and 1-3 degrees for rotation. Modification of the threshold values was necessary in 7 cases of the input images, hence the default threshold values were correct in $80 \%$ of the patient image pairs. Due to the possibility of the threshold value modifications it was possible to detect all epileptic foci of all patients.

\section{Conclusion}

The most trivial way of detecting hop spots inside the brain is subtracting the interictal image from the ictal one, although this step generates many false positive spots in the subtraction hence the multiple examination of the subtraction and ictal image is necessary. Since our method excludes the normal brain area from the visualization by building on the fact that hotspots always have a high activity, the number of false positive hot spots occurrences in the result is minimized. Due to the possibility of modifying the threshold values our tool is flexible enough to generate a specific result for all the patients. Our normalization step operates with local brain values instead of global ratios between the images. Due to the local normalization the global grey value stretching of the images is avoided which would not lead to a sophisticated normalization [2]. The clinical evaluation of our method is still under investigation, since higher number of inputs is needed, although based on our present 35 patient data the first results show that our program may become a useful tool for epileptic patient investigations.

\section{References}

1. Koo CW, Devinsky O, Hari K, et al. Stratifying differences on ictal/interictal subtraction SPECT images. Epilepsia. 2003;44(3):379-386.

2. Radau PE, Slomka PJ, Julin P, et al. Evaluation of linear registration algorithms for brain SPECT and the errors due to hypoperfusion lesions. Med Phys. 2001;28:16601668.

3. Boussion N, Houzard C, Ostrowsky K, et al. Automated detection of local normalization areas for ictal-interictal brain SPECT. J Nucl Med. 2002;43(11):1419-1425.

4. Pluim JPW, Maintz JBA, Viergever MA. Mutual-information-based registration of medical images: A survey. IEEE Trans Med Imaging. 2003;22(8):986-1004.

5. Bernon JL, Mariano-Goulart D, Zanca A. A comparative study of Powell's and Downhill simplex algorithms for a fast multimodal surface matching in brain imaging. Comput Med Imaging Graph. 2001;25(4):287-297.

6. Peng J, Rusch PH, Herrmann T, et al. Morphological filters and edge detection application to medical imaging. Procs IEEE EMBS. 1991;13:251-252. 